\title{
Exploring absolute and relative measures of exposure to food environments in relation to dietary patterns among European adults
}

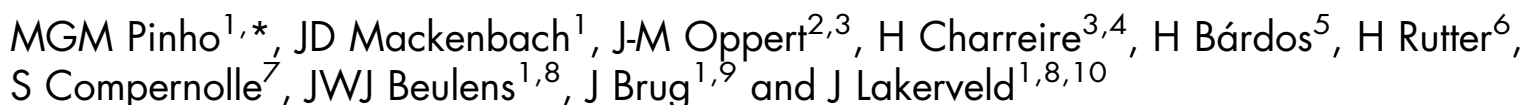

${ }^{1}$ Amsterdam UMC, Vrije Universiteit Amsterdam, Department of Epidemiology and Biostatistics, Amsterdam Public Health, De Boelelaan 1089a, Amsterdam, The Netherlands: ${ }^{2}$ Sorbonne Université, Institute of Cardiometabolism and Nutrition, Department of Nutrition, Paris, France: ${ }^{3}$ Equipe de Recherche en Epidémiologie Nutritionnelle (EREN), Centre de Recherche en Epidémiologie et Statistiques, Inserm (U1 153), Inra (U1 125), Cnam, COMUE Sorbonne Paris Cité, Université Paris 13, Bobigny, France: ${ }^{4}$ Université Paris Est, Lab-Urba, UPEC, Créteil, France: ${ }^{5}$ Department of Preventive Medicine, Faculty of Public Health, University of Debrecen, Debrecen, Hungary: ${ }^{6}$ Department of Social and Policy Sciences, University of Bath, Bath, UK: ${ }^{7}$ Department of Movement and Sport Sciences, Faculty of Medicine and Health Sciences, Ghent University, Ghent, Belgium: ${ }^{8}$ Julius Center for Health Sciences and Primary Care, University Medical Center Utrecht, Utrecht, The Netherlands: ${ }^{9}$ Amsterdam School of Communication Research (ASCoR), University of Amsterdam, Amsterdam, The Netherlands: ${ }^{10}$ Faculty of Geosciences, Utrecht University, Utrecht, The Netherlands

Submitted 18 March 2018: Final revision received 19 September 2018: Accepted 2 October 2018: First published online 7 December 2018

\begin{abstract}
Objective: To explore the associations of absolute and relative measures of exposure to food retailers with dietary patterns, using simpler and more complex measures.

Design: Cross-sectional survey.

Setting: Urban regions in Belgium, France, Hungary, the Netherlands and the UK. Participants: European adults ( $n$ 4942). Supermarkets and local food shops were classified as 'food retailers providing healthier options'; fast-food/takeaway restaurants, cafés/bars and convenience/liquor stores as 'food retailers providing less healthy options'. Simpler exposure measures used were density of healthy and density of less healthy food retailers. More complex exposure measures used were: spatial access (combination of density and proximity) to healthy and less healthy food retailers; density of healthier food retailers relative to all food retailers; and a ratio of spatial access scores to healthier and less healthy food retailers. Outcome measures were a healthy or less healthy dietary pattern derived from a principal component analysis (based on consumption of fruits, vegetables, fish, fast foods, sweets and sweetened beverages).

Results: Only the highest density of less healthy food retailers was significantly associated with the less healthy dietary pattern $(\beta=-129 \cdot 6$; 95\% CI $-224 \cdot 3$, $-34.8)$. None of the other absolute density measures nor any of the relative measures of exposures were associated with dietary patterns.

Conclusions: More complex measures of exposure to food retailers did not produce stronger associations with dietary patterns. We had some indication that absolute and relative measures of exposure assess different aspects of the food environment. However, given the lack of significant findings, this needs to be further explored.
\end{abstract}

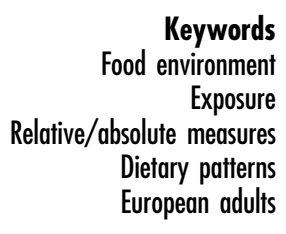

Abundant availability of foods in general and high accessibility to high-energy (ultra-processed) foods high in salt, sugar and/or saturated fat characterize current food environments worldwide ${ }^{(1)}$. Promoting healthier food environments through policy actions may contribute to healthier eating and consequently to obesity prevention at the population level ${ }^{(2-4)}$.

There is evidence supporting a link between the residential food environment - the distribution of food retailers within an individual's residential area - and dietary 
behaviours $^{(5-7)}$, but the evidence is inconsistent. A systematic review from Caspi et al. showed that the density of food retailers was significantly associated with dietary intake in thirteen out of the twenty studies reviewed. Studies using the distance to food retailers provide less consistent results: seven out of thirteen studies found no significant associations with dietary intake and two studies presented mixed results ${ }^{(8)}$. The review from Bivoltsis et al. also found that density measures produce more consistent effect sizes than distance measures; they recommended future studies to follow a multi-method approach where ideally a combination of measures is used to assess both availability and accessibility ${ }^{(9)}$.

The assessment of exposure to the neighbourhood food environment is complex and context-dependent ${ }^{(10)}$. In studies on exposure to food retailers in relation to diet, measures of geographic accessibility (i.e. proximity) and measures of geographic availability (i.e. density) are most often used $^{(8,11)}$. A potential limitation of assessing exposure to food retailers using simpler measures (e.g. only proximity or density) is that it may not reflect the complexity of exposure to food retailers. For instance, if the distance of two study participants to their nearest food retailer is similar but the density of retailers in their neighbourhood is very different, the use of a proximitybased measure such as distance would not fully capture the difference in exposure. Therefore, while both measures may be relevant to define exposure, a combination of measures (e.g. an absolute measure that accounts for both density and proximity or a relative measure that accounts for more than one type of food retailer) may be a better option. For example, Salze et al. used the estimation of spatial accessibility based on a 'potential accessibility index' that encompasses functions of the weighted inverse distance to destinations within a specific area, as such allowing to take account of both proximity and availability $^{(12)}$. The spatial accessibility measure was used before to explore the relation between fast-food access and body weight ${ }^{(13)}$.

Another potential limitation of many studies on the food environment is a focus on absolute measures of exposure, namely exposure to only one type of food retailer while ignoring the relative influence of a variety of food retailers ${ }^{(14,15)}$. For instance, if only density of fast-food restaurants in a neighbourhood is considered without accounting for the presence of other types of food retailers, results might be biased because access to healthier food retailers may balance the influence that less healthy food retailers have on dietary choices ${ }^{(16)}$. Therefore, it has been argued that the use of relative measures which take into account the variety of food retailers within the broader food environment might be preferable ${ }^{(8,16-20)}$. An example of a relative measure is the modified Retail Food Environment Index (mRFEI), representing the percentage of healthier food retailers relative to the total amount of food retailers in the area ${ }^{(21)}$. While this measure was originally developed in the USA, it is likely that the ratio of healthier and unhealthier options in the food environment is of relevance in a European context as well. Moreover, the majority of studies on the relationship between the food environment and diet have been conducted in the USA, Australia and New Zealand ${ }^{(8)}$, highlighting the need for studies of the food environment in a European context.

From the dietary perspective, most studies analysing the association between the food environment and diet have focused on the consumption of specific foods, for instance fruits and vegetables or fast-food meals ${ }^{(6,16,22,23)}$. However, an individual's diet consists of multiple components, which may all be influenced by the food environment. As such, in the present study we used respondents' reported consumption of different foods and drinks to derive latent (a posteriori) dietary patterns using principal component analysis. This is a widely used method for the analysis of dietary data that is applied to obtain data reduction by grouping highly correlated food variables into components ${ }^{(24-26)}$.

We aimed to test the associations of absolute and relative measures of exposure to food retailers with dietary patterns, using simpler and more complex measures. We hypothesized that: (i) more complex absolute measures, such as spatial accessibility, provide stronger associations with dietary patterns than simpler absolute measures; and (ii) relative measures of exposure to food retailers produce stronger associations with dietary patterns than when absolute measures of exposure are used.

\section{Methods}

\section{Study design, sampling and participants}

The current study was part of the European SPOTLIGHT project $^{(27)}$. A web-based survey was conducted in five European urban regions: Ghent and suburbs (Belgium), Paris and inner suburbs (France), Budapest and suburbs (Hungary), the Randstad (a conurbation including the cities of Amsterdam, Rotterdam, The Hague and Utrecht in the Netherlands) and Greater London (UK). We randomly sampled twelve neighbourhoods in each urban region, based on a combination of residential density and socioeconomic status (SES) data at the neighbourhood level. This resulted in four pre-specified neighbourhood types: low SES/low residential density, low SES/high residential density, high SES/low residential density and high SES/ high residential density. Three neighbourhoods of each type were randomly sampled (i.e. twelve per country, sixty in total). Neighbourhoods were defined as the smallest-scale local administrative boundaries for all countries except for Hungary. The administrative boundaries for Budapest region were much larger and more heterogeneous compared with the other regions under study; therefore, to make neighbourhoods comparable across countries, we defined one square kilometre $\left(1 \mathrm{~km}^{2}\right)$ 
area as the study area in Hungary. Mean neighbourhood sizes ranged from $0.3 \mathrm{~km}^{2}$ in France to $3.6 \mathrm{~km}^{2}$ in the UK. Most of the participants ( $n$ 4942) were from Ghent (32.2\%) and the fewest participants were from the UK (10.6\%). Detailed descriptions of the neighbourhoods' characteristics and sampling, study design and participant recruitment have been provided elsewhere ${ }^{(28)}$.

Between February and September 2014, individuals aged 18 years or older living in the selected neighbourhoods were invited to participate in an online survey regarding the food and physical activity environments. The questionnaire included questions on demographics, residential neighbourhood perceptions, social environmental factors, health, motivations for and barriers to engaging in healthy behaviours, dietary behaviours, and self-reported weight and height. A total of 6037 individuals participated in the study (10.8\% of the 55893 invited). Local ethics committees in each participating country approved the study. All survey participants provided informed consent.

\section{Measures}

Exposure to the food environment (independent variables) The food environment in the selected residential neighbourhoods was objectively measured using the validated SPOTLIGHT Virtual Audit Tool (SPOTLIGHT-VAT) from February to September 2014. More information about the psychometric properties of the tool can be found elsewhere $^{(29)}$. Briefly, the intra-observer reliability ranged from $92 \%$ agreement $(\kappa=0.65)$ to $100 \%$ agreement $(\kappa=1 \cdot 00)$, and the inter-observer reliability ranged from $79 \%$ agreement $(\kappa=0.44)$ to $99 \%$ agreement $(\kappa=0.58)$. All street segments from fifty-seven residential neighbourhoods were virtually audited; three neighbourhoods were excluded from the analysis because they were not covered by Google Street View at the time of data collection or had no food retailers present. During the audit, researchers rated a total of 4482 street segments on forty-two items representing eight dimensions of the food and physical activity environments. In addition, we stored geographical coordinates as well as the type of each food outlet in a Geographical Information System.

The food retailers analysed in the present study were supermarkets, local food shops (such as butchers and bakeries), fast-food/takeaway restaurants, cafés/bars and convenience/liquor stores (this category includes convenience stores that may or may not sell alcohol and stores that sell alcohol only). Classifying food retailers according to their healthfulness is not a straightforward procedure and there is no clear definition on the healthiness of food retailers. Even though the relationship between access to supermarkets and healthier dietary habits is not fully understood, supermarkets are often considered a source of healthy foods ${ }^{(30)}$. The literature shows less consensus when it comes to access to restaurants: while eating away from home has been associated with lower diet quality, especially increased total energy, and both higher fat and lower micronutrient intakes ${ }^{(31,32)}$, full-service restaurants and fast-food restaurants might play different roles on diet and health outcomes ${ }^{(33,34)}$. Therefore, we considered fastfood/takeaway restaurants, cafés/bars and convenience/ liquor stores as food retailers providing less healthy options'. We also performed a sensitivity analysis including full-service restaurants in the latter category. We considered supermarkets and local food shops as food retailers providing more healthy options'. For the sake of simplicity, in the present paper these categories are referred to as 'less healthy food retailers' and 'healthier food retailers', respectively. Using ArcGIS version 10.4, all food retailers within the limits of each individual's residential neighbourhood (defined using administrative boundaries) plus a $300 \mathrm{~m}$ Euclidian buffer around it were geo-localised. We added the $300 \mathrm{~m}$ Euclidian buffer around the neighbourhoods to also capture a potentially relevant neighbourhood area for those living near the border of the administrative neighbourhood. An example of how this buffer around the administrative neighbourhoods was constructed is given in the Fig. 1.

To understand how different measures of exposure to the food environment were related to dietary patterns, we created six exposure variables. First, based on a common measure of exposure described in the literature ${ }^{(11)}$, we created two absolute density-based measures: density of healthier food retailers and density of less healthy food retailers per square kilometre. Then, we calculated two spatial access scores: spatial access to healthier and less healthy food retailers. Spatial access measures take account of both proximity and availability of food retailers. To compute this measure, the Euclidian distances from the participant's address to each food retailer in the neighbourhood are calculated (which accounts for proximity measures). By summing the inverse distances calculated for the different food retailers in the neighbourhood, availability is also taken into account. In this way, the more food retailers are present in a neighbourhood, the more (inverse) distances will be summed. Therefore, the highest spatial access score is assigned to an individual living at the shortest distance to the highest number of food retailers. In addition, to obtain a density-based relative indicator, we calculated the $\mathrm{mRFEI}^{(21)}$ which represents the proportion of healthier food retailers in relation to the total amount of food retailers in the neighbourhood. Finally, to obtain a relative indicator based on proximity, density and variety, we created a ratio for the spatial access scores where individuals with a higher ratio score have a higher access to healthier food retailers relative to the total amount of food retailers in the neighbourhood. The equations used for calculating the exposure measures as well as a classification of the measures into 'simpler absolute measures', 'complex absolute measures' and 'complex relative measures' are presented in Table 1. 


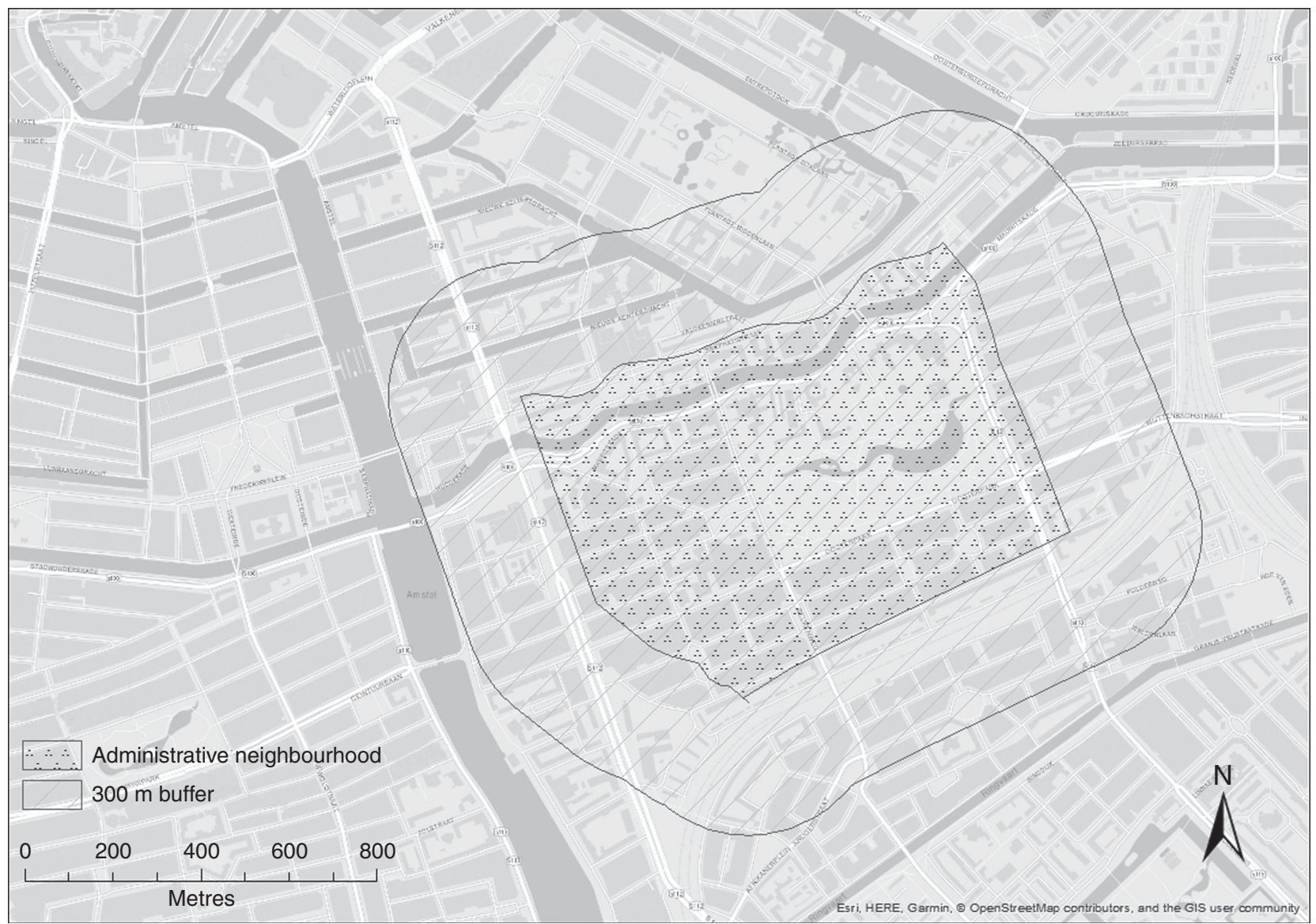

Fig. 1 Example of how the $300 \mathrm{~m}$ Euclidean buffer around the administrative neighbourhoods was constructed using data for Oosterparkbuurt, the Netherlands

Table 1 Description and classification of the exposure measures used in the SPOTLIGHT project

\begin{tabular}{|c|c|c|}
\hline \\
\hline \multicolumn{3}{|l|}{$\begin{array}{l}\text { Simpler absolute measures } \\
\text { Density of healthier food retailers }\end{array}$} \\
\hline Density of less healthy food retailers & $=$ & $\frac{\text { No. of less healthy food retailers }}{\text { Neighbourbood area }\left(\mathrm{km}^{2}\right)}$ \\
\hline Complex absolute measures & & \\
\hline Spatial access to healthier food retailers & $=$ & $\sum_{\frac{\text { Euclidian distance to healthier food retailers }{ }_{n}}{1}}$ \\
\hline Spatial access to less healthy food retailers & $=$ & $\sum \frac{1}{\text { Euclidian distance to less healthy food retailers }}$ \\
\hline $\begin{array}{l}\text { Complex relative measures } \\
\text { Modified Retail Food Environment Index (mRFEI) }\end{array}$ & $=$ & $100 \times \frac{\text { No. of healthier food retailers }}{\text { No. of healthier food retailers }+ \text { no. of less healthy food retailers }}$ \\
\hline Ratio of spatial access scores & $=$ & $100 \times \frac{\text { Spatial access score to healthier food retailers }}{\text { Spatial access score to bealthier + less healthy food retailers }}$ \\
\hline
\end{tabular}

As the units of measurement across the measures used are different, and the independent variables were not normally distributed, we split the independent variables into tertiles to increase comparability.

\section{Dietary patterns (outcome variables)}

The weekly frequency of consumption of fruits, vegetables, fish, fast foods, sweets and sweetened beverages was assessed through the online survey by asking the following question for each of the items: 'How many times a week do you eat fruits, vegetables, fish, [...]?' There were nine response options ranging from 'once a week or less' to 'more than twice a day'.

Due to the non-normal distribution, the dietary variables were square-root-transformed. To identify common patterns of food consumption, we performed principal component analysis of the square-root-transformed variables using the correlation matrix and varimax rotation. By observing the Kaiser criterion (eigenvalue greater than 1), the scree plot and the interpretability of components, we decided to retain two principal components. Food items with an absolute factor loading greater than 0.30 were 
considered part of the corresponding component. The first rotated component was composed of fruits (factor loading $=0.79)$, vegetables $(0.81)$ and fish $(0.55)$; and the second rotated component was composed of sweets $(0.75)$, sweetened beverages $(0.70)$ and fast foods $(0.40)$. The total variance explained by these two components was $47.6 \%$. Scores reflecting the weighted values of each food item in each of the respective components were then assigned to individuals ${ }^{(24-26)}$. The first component was named 'healthy dietary pattern' and the second component was named 'less healthy dietary pattern'. These are standardized scores (mean of 0 and SD of 1). Since the scores were centred around 0 , the interquartile range (IQR) for the healthy dietary pattern was -0.6 to 0.5 ; and the IQR for the less healthy dietary pattern was -0.8 to 0.6 . These scores were multiplied by 1000 to enhance interpretation of the regression coefficients. Therefore, a 1000point change in the outcome would represent a change equivalent to $1 \mathrm{sD}$. A higher score on the "healthy dietary pattern' represents a higher frequency of consumption of foods on this pattern, namely fruits, vegetables and fish. A higher score on the 'less healthy dietary pattern' represents a higher frequency of consumption of foods on this pattern, namely fast foods, sweets and sweetened beverages.

\section{Covariates}

We adjusted for participants' age, sex, educational attainment, household composition (total number of adults and children) and urban region. Educational systems differ across countries, so the educational attainment variable was categorized into two groups that were internationally comparable: 'lower' (secondary education or less) and 'higher' (college or university level) education. Household composition was categorized into three groups: 'one adult, no child', 'two adults, no child' and 'adult(s) and child(ren)'. To minimize bias resulting from residential selfselection $^{(35)}$, we adjusted for three variables related to neighbourhood choice. We asked participants if the presence of restaurants was a factor that influenced their decision to live in their current neighbourhood; this variable was named 'preference for restaurants in the neighbourhood' (no/yes). We also asked whether participants spent most of their spare time in their residential neighbourhood (no/yes) and about their duration of residency ('less than 10 years'/'10 years or more'). We also present a sensitivity analysis unadjusted for the self-selection variables.

\section{Statistical analysis}

Individuals whose residential addresses were located outside the selected neighbourhoods were excluded from the analysis. We also excluded individuals living in a neighbourhood where Google Street View and geolocalisation of food retailers was not possible during the time of data collection. Individuals living in neighbourhoods without any food retailers were also excluded since it would not be possible to calculate the relative measure of exposure for them. In total 1095 participants were excluded, resulting in a final analytical sample of 4942 respondents distributed over fifty-seven neighbourhoods. We handled missing data under the assumption that data were missing at random by applying multiple imputation via predictive mean matching to all variables in the analysis, namely outcome, determinants and covariates ${ }^{(36)}$. As recommended by Rubin ${ }^{(37)}$ and Bodner ${ }^{(38)}$, the missing value patterns in our data set were evaluated showing that the percentage of missing values ranged from $1 \%$ (age) to $30 \cdot 2 \%$ (preference for restaurants in the neighbourhood). For this reason, we chose to impute thirty data sets.

We performed descriptive statistics using non-imputed data. To examine the extent to which the six different measures of exposure to the food environment were unique, we calculated Pearson's correlation coefficients. Pooled results from imputed data were used to answer our research questions. We built six different models to test the association between the six different exposure measures (density of healthier and density of less healthy food retailers; spatial access to healthier and spatial access to less healthy food retailers; mRFEI and ratio of spatial access scores) and the outcomes healthy and less healthy dietary patterns. Due to the clustered nature of our data, we performed linear models using generalized estimating equations with an exchangeable structure and with the neighbourhoods as a grouping variable ${ }^{(39)}$. All models were a priori adjusted for age, sex, educational attainment, household composition, urban region and the three variables related to neighbourhood choice. Statistical significance was determined at an $\alpha$ level of $5 \%$.

Descriptive and multilevel linear regression analyses, as well as the multiple imputation procedure, were performed using the statistical software package Stata ${ }^{\circledR}$ version 14.1. Spatial analyses were conducted in ArcGIS version 10.4 .

\section{Results}

Table 2 describes the characteristics of participants. The median density of food retailers providing more healthy options was 1.42 per $\mathrm{km}^{2}$ (IQR 0.68-6.19) and with a median of 2.31 food retailers per $\mathrm{km}^{2}$ (IQR 0.60-9.56), the density of food retailers providing less healthy options was much higher. The mRFEI indicated that the median proportion of healthier food retailers in relation to all food retailers in the neighbourhoods was $49.49 \%$. The median score for spatial access to healthier food retailers was 0.009 (IQR 0.004-0.36) and for spatial access to less healthy food retailers was 0.008 (IQR 0.001-0.044). The ratio for the spatial access scores indicating the relative median proportion of healthier food retailers in the neighbourhoods 
Table 2 Descriptive characteristics of the participants: adults in neighbourhoods from five urban regions in Europe, February-September 2014. The SPOTLIGHT project ( $n$ 4942)

\begin{tabular}{|c|c|c|c|}
\hline & $n$ & Mean, \% or median & SD or IQR \\
\hline Age (years), mean and SD & 4893 & $52 \cdot 3$ & $16 \cdot 4$ \\
\hline Sex $(\%)$ & 4893 & & \\
\hline Female & & 55.5 & \\
\hline Educational attainment (\%) & 4470 & & \\
\hline Higher & & $54 \cdot 1$ & \\
\hline Household composition (\%) & 4471 & & \\
\hline 1 adult, no children & & $22 \cdot 4$ & \\
\hline 2 adults, no children & & $47 \cdot 8$ & \\
\hline Adult(s) with child(ren) & & $29 \cdot 8$ & \\
\hline Urban regions (\%) & 4942 & & \\
\hline Ghent and suburbs (Belgium) & & $34 \cdot 2$ & \\
\hline Paris and suburbs (France) & & $14 \cdot 3$ & \\
\hline Budapest and suburbs (Hungary) & & $14 \cdot 4$ & \\
\hline The Randstad (the Netherlands) & & $26 \cdot 6$ & \\
\hline Greater London (the UK) & & $10 \cdot 6$ & \\
\hline Duration of residency in this neighbourhood (\%) & 4741 & & \\
\hline Less than 10 years & & $35 \cdot 0$ & \\
\hline 10 years or more & & $65 \cdot 0$ & \\
\hline Spare time spent in the neighbourhood (\%) & 4801 & & \\
\hline Yes & & 71.8 & \\
\hline Preference for restaurants in the neighbourhood (\%) & 3449 & & \\
\hline Yes & & $16 \cdot 2$ & \\
\hline Density of healthier food retailers, median and IQR* & 4942 & 1.42 & $0.68-6 \cdot 19$ \\
\hline Density of less healthy food retailers, median and IQR* & 4942 & $2 \cdot 31$ & $0.60-9.56$ \\
\hline mRFEI, median and IQR $\dagger$ & 4942 & 49.49 & $32 \cdot 50-62 \cdot 50$ \\
\hline Spatial access to healthier food retailers, median and IQR & 4942 & 0.009 & $0.004-0.036$ \\
\hline Spatial access to less healthy food retailers, median and IQR & 4942 & 0.008 & $0.001-0.044$ \\
\hline Ratio for the spatial access scores, median and IQR§ & 4942 & 46.35 & $28 \cdot 69-84 \cdot 61$ \\
\hline Score for healthy dietary pattern, mean and SD $\|$ & 3950 & 0 & 1000 \\
\hline Score for less healthy dietary pattern, mean and SD $\|$ & 3950 & 0 & 1000 \\
\hline \multicolumn{4}{|c|}{$\begin{array}{l}\text { IQR, interquartile range; mRFEI, modified Retail Food Environment Index. } \\
\text { 'Density represents the count of food retailers divided by the neighbourhood area in square kilometres. } \\
\text { tmRFEI represents the proportion of healthier food retailers in relation to the total number of food retailers in the neighbourhood. } \\
\text { fSpatial access score represents an inverse function of the sum of the calculated distances from individuals' home address to each } \\
\text { healthier and less healthy food outlet in the residential neighbourhood. } \\
\text { §Ratio for the spatial access scores represents spatial access scores to healthier food retailers divided by healthier plus less healthy } \\
\text { food retailers. } \\
\| \text { Scores for healthier and less healthy dietary patterns were multiplied by } 1000 \text {. }\end{array}$} \\
\hline
\end{tabular}

was $46 \cdot 35 \%$. The median density score for the healthier dietary pattern was -85.9 (IQR $-591 \cdot 1,510 \cdot 6$ ) and for the less healthy dietary pattern was $-63 \cdot 6$ (IQR -767·6, 583.1; data not shown).

Table 3 shows the Pearson correlation coefficients for the six exposure measures. The density of healthier food retailers was strongly associated with the density of less healthy food retailers $(r=0 \cdot 79)$. Spatial access to healthier food retailers was strongly associated with the density of both healthier $(r=0.84)$ and less healthy $(r=0.75)$ food retailers, while this was not the case for the densities and spatial access to less healthy food retailers $(r=0.15$ and $r=0 \cdot 26$, respectively). Differently from the density measures, the two spatial access measures were not strongly associated $(r=0 \cdot 16)$. While the ratio of spatial access was strongly associated to the mRFEI $(r=0 \cdot 87)$, it was not strongly associated to the other four absolute exposure measures.

Table 4 shows the associations between the absolute measures of exposure and both healthy and less healthy dietary patterns. The highest density of less healthy food retailers was significantly associated with a lower score on the less healthy dietary pattern. The effect size, however, is rather small considering that the outcome measure was multiplied by 1000 to enhance interpretation of the results ( $\beta=-129 \cdot 6 ; 95 \%$ CI $-224 \cdot 3,-34 \cdot 8)$.

Table 5 shows the associations between the relative measures of exposure and the dietary patterns. We did not find any significant association with the dietary patterns using either of the relative measures tested, and the effect sizes are negligible.

The online supplementary material, Supplementary Tables 1 and 2, shows the results from sensitivity analyses where we included full-service restaurants in the category of less healthy food retailers. These results are highly comparable to the main analyses where full-service restaurants were left out. In Supplementary Table 1, the most notable difference is that the association between density of less healthy food retailers and less healthy dietary patterns was no longer significant when full-service restaurants were included in the less healthy category. In addition, although still not statistically significant and with very small effect sizes, the direction of the association between spatial access to less healthy food retailers and 
Table 3 Pearson correlation coefficients for the six measures of exposure to the food environment among adults in neighbourhoods from five urban regions in Europe, February-September 2014. The SPOTLIGHT project $(n$ 4942)

\begin{tabular}{|c|c|c|c|c|c|c|}
\hline & $\begin{array}{l}\text { Density of } \\
\text { healthier food } \\
\text { retailers }\end{array}$ & $\begin{array}{l}\text { Density of less } \\
\text { healthy food } \\
\text { retailers }\end{array}$ & mRFEI & $\begin{array}{l}\text { Spatial access to } \\
\text { healthier food } \\
\text { retailers }\end{array}$ & $\begin{array}{l}\text { Spatial access to less } \\
\text { healthy food retailers }\end{array}$ & $\begin{array}{l}\text { Ratio of spatial } \\
\text { access scores }\end{array}$ \\
\hline $\begin{array}{l}\text { Density of healthier } \\
\text { food retailers }\end{array}$ & 1 & & & & & \\
\hline $\begin{array}{l}\text { Density of less healthy } \\
\text { food retailers }\end{array}$ & $0 \cdot 7884$ & 1 & & & & \\
\hline mRFEI & -0.0486 & -0.2999 & 1 & & & \\
\hline $\begin{array}{l}\text { Spatial access to } \\
\text { healthier food } \\
\text { retailers }\end{array}$ & 0.8418 & 0.7475 & -0.0952 & 1 & & \\
\hline $\begin{array}{l}\text { Spatial access to less } \\
\text { healthy food retailers }\end{array}$ & $0 \cdot 1472$ & $0 \cdot 2551$ & -0.0879 & $0 \cdot 1649$ & 1 & \\
\hline $\begin{array}{l}\text { Ratio of spatial access } \\
\text { scores }\end{array}$ & 0.0290 & -0.2376 & 0.8720 & 0.0368 & -0.1432 & 1 \\
\hline
\end{tabular}

mRFEl, modified Retail Food Environment Index.

Table 4 Coefficients and $95 \% \mathrm{Cl}$ as derived from generalized estimating equation-linear regression analyses indicating the associations of absolute measures of exposure to food retailers with dietary patterns among adults in neighbourhoods from five urban regions in Europe, February-September 2014. The SPOTLIGHT Project $(n$ 4942)

\begin{tabular}{|c|c|c|c|c|c|c|}
\hline & \multicolumn{3}{|c|}{ Healthy dietary pattern ${ }^{*} \dagger$} & \multicolumn{3}{|c|}{ Less healthy dietary pattern†‡ } \\
\hline & Coefficient & $95 \% \mathrm{Cl}$ & $P$ value & Coefficient & $95 \% \mathrm{Cl}$ & $P$ value \\
\hline \multicolumn{7}{|c|}{ Tertiles of density of healthier food retailers per $\mathrm{km}^{2} \S$} \\
\hline Lowest & 1 & & & 1 & & \\
\hline Medium & $-48 \cdot 2$ & $-233 \cdot 6,137.3$ & 0.611 & 13.7 & $-98 \cdot 1,125 \cdot 5$ & 0.810 \\
\hline Highest & -10.5 & $-178 \cdot 8,157 \cdot 8$ & 0.903 & $-56 \cdot 6$ & $-163 \cdot 0,49 \cdot 9$ & 0.297 \\
\hline \multicolumn{7}{|c|}{ Tertiles of density of less healthy food retailers per $\mathrm{km}^{2} \|$} \\
\hline Lowest & 1 & & & 1 & & \\
\hline Medium & $-78 \cdot 1$ & $-244 \cdot 9,88.8$ & 0.359 & -61.3 & $-176 \cdot 3,53 \cdot 7$ & 0.296 \\
\hline Highest & $-23 \cdot 3$ & $-181 \cdot 3,122 \cdot 7$ & 0.706 & -129.6 & $-224 \cdot 3,-34 \cdot 8$ & 0.007 \\
\hline \multicolumn{7}{|c|}{ Tertiles of spatial access score for healthier food retailers§ } \\
\hline Lowest & 1 & & & 1 & & \\
\hline Medium & $-77 \cdot 2$ & $-183 \cdot 1,28 \cdot 7$ & $0 \cdot 153$ & $-18 \cdot 2$ & $-115.5,79.2$ & 0.714 \\
\hline Highest & -99.7 & $-222 \cdot 9,23 \cdot 5$ & 0.113 & -34.4 & $-133 \cdot 8,65 \cdot 0$ & 0.498 \\
\hline \multicolumn{7}{|c|}{ Tertiles of spatial access score for less healthy food retailers\| } \\
\hline Lowest & 1 & & & 1 & & \\
\hline Medium & -68.0 & $-164 \cdot 9,28.9$ & 0.169 & -6.67 & $-90 \cdot 9,77.6$ & 0.876 \\
\hline Highest & $-100 \cdot 0$ & $-224 \cdot 3,24 \cdot 2$ & 0.114 & $-9 \cdot 00$ & $-117 \cdot 4,99 \cdot 4$ & 0.871 \\
\hline
\end{tabular}

Dietary patterns were obtained from principal component analysis. All models were adjusted for age, sex, educational attainment, household composition, urban region and self-selection variables.

${ }^{\star}$ Healthy dietary pattern is composed of fruits, vegetables and fish.

tScores for healthy and less healthy dietary patterns were multiplied by 1000

†Less healthy dietary pattern is composed of fast foods, sweets and sweetened beverages.

$\S$ Healthier food retailers: supermarkets and local shops;

IILess healthy food retailers: fast-food restaurants, cafés/bars and convenience/liquor stores.

the less healthy dietary pattern changed: the previously negative association became positive when full-service restaurants were considered. In contrast, in Supplemental Table 2, which shows the association for relative measures, the previously positive association between the ratio for spatial access scores and less healthy dietary patterns became negative when full-service restaurants were included in the less healthy food retailers. The effect sizes, in turn, became even smaller. Supplemental Tables 3 and 4 show the results from sensitivity analyses where we did not adjust our models for the self-selection variables. Results from these analyses are comparable to the main analyses which are adjusted for self-selection.

\section{Discussion}

We tested the association of different neighbourhood food exposure measures with dietary patterns. We aimed to provide a comprehensive picture of these potential associations by using both simpler and more complex measures of exposure. We used density and spatial access scores (with the latter accounting for both density and proximity) and also relative measures of exposure to food retailers, namely the mRFEI and a ratio of spatial access scores to healthier and less healthy food retailers.

The correlation analyses showed important differences across the exposure measures used. The fact that density 
Table 5 Coefficients and $95 \% \mathrm{Cl}$ as derived from generalized estimating equation-linear regression analyses indicating the associations of relative measures of exposure to food retailers with dietary patterns among adults in neighbourhoods from five urban regions in Europe, February-September 2014. The SPOTLIGHT Project $(n$ 4942)

\begin{tabular}{|c|c|c|c|c|c|c|}
\hline & \multicolumn{3}{|c|}{ Healthy dietary pattern ${ }^{*} \dagger$} & \multicolumn{3}{|c|}{ Less healthy dietary pattern†‡ } \\
\hline & Coefficient & $95 \% \mathrm{Cl}$ & $P$ value & Coefficient & $95 \% \mathrm{Cl}$ & $P$ value \\
\hline \multicolumn{7}{|c|}{ Tertiles of the mRFEI } \\
\hline Lowest & 1 & & & 1 & & \\
\hline Medium & -28.4 & $-231 \cdot 2,174 \cdot 4$ & 0.784 & $13 \cdot 1$ & $-107 \cdot 2,133 \cdot 4$ & 0.831 \\
\hline Highest & 86.8 & $-88 \cdot 7,262 \cdot 4$ & 0.332 & 93.4 & $-9.0,195.7$ & 0.074 \\
\hline \multicolumn{7}{|c|}{ Tertiles of the ratio for spatial access scores } \\
\hline Lowest & 1 & & & 1 & & \\
\hline Medium & $-58 \cdot 8$ & $-173.5,67.9$ & 0.391 & $20 \cdot 4$ & $-69 \cdot 6,110 \cdot 4$ & 0.657 \\
\hline Highest & 48.0 & $-94.5,190.5$ & 0.509 & $36 \cdot 0$ & $-65 \cdot 0,137 \cdot 1$ & 0.484 \\
\hline
\end{tabular}

mRFEI, modified Retail Food Environment Index.

Dietary patterns were obtained from principal component analysis. All models were adjusted for age, sex, educational attainment, household composition, urban region and self-selection variables.

*Healthy dietary pattern is composed of fruits, vegetables and fish.

†Scores for healthy and less healthy dietary patterns were multiplied by 1000 .

†Less healthy dietary pattern is composed of fast foods, sweets and sweetened beverages.

of healthier food retailers was strongly correlated with the density of less healthy food retailers suggests that healthier and less healthy food retailers often co-locate ${ }^{(40)}$. Spatial access to healthier food retailers, but not spatial access to less healthy food retailers, was associated with both densities. The spatial access score is a more tailored measure than only density because in its calculation the weighted distances to food retailers are derived and summed up which then accounts for both density and proximity. However, when there are few food retailers in the neighbourhood, the spatial access measure will be very similar to the density measure, as only a few distances will be added in the calculation. Therefore, because in the present study there was on average a higher number of less healthy food retailers than healthier ones, the spatial access to less healthy food retailers was not strongly related to either of the two density measures. This may also explain why the two spatial access measures were not strongly correlated. Not surprisingly, the ratio of spatial access was strongly correlated to the mRFEI, as both are relative measures accounting for the ratio of healthier food retailers to the total amount of food retailers in a neighbourhood. On the other hand, the two ratio measures were not strongly correlated to the four absolute exposure measures, confirming that absolute and relative measures assess different aspects of the food environment.

The density of less healthy food retailers was associated with lower scores on the less healthy dietary pattern. This negative association is unexpected. However, it is interesting to note that all associations from Table 3, which presents the associations using the absolute measures of exposure, are related to lower scores on any of the dietary patterns - although effect sizes are all very small and nonsignificant. In contrast, in Table 4, which presents the associations using the relative measures of exposures, most measures of exposure are related to higher scores on both dietary patterns. Considering the lack of associations and the very small effect sizes, we cannot confirm our hypothesis that more complex and relative exposure measures would be more consistently related to dietary patterns than simpler measures. Nevertheless, the observed pattern with the direction of the associations across Tables 3 and 4 reinforces the conclusion obtained from the correlation analysis (Table 2) on the different nature of absolute and relative measures of exposure.

We could not demonstrate that relative measures of exposure could provide more consistent associations between the food environment and diet, yet there has been a growing body of literature indicating that ${ }^{(16,41,42)}$. There is evidence suggesting that despite the presence of less healthy food retailers in the neighbourhood might encourage the consumption of unhealthier foods, the concomitant presence of healthier options may reduce the potential harmful effect on individuals' diets ${ }^{(16)}$. However, as our results indicate, at best, that the choice of exposure measure has an impact on the findings, the reasons for the adjustment for the broader food environment using relative measures (that typically account for the presence of both healthy and less healthy food retailers) should be made explicit and discussed. For instance, it may be that adjustment for the broader food environment is only relevant when the food retailers considered are of a similar type, for example healthier and less healthy dining options or healthier and less healthy food stores. On this matter, Polsky et al. found that a higher presence of fast-food restaurants relative to the presence of other restaurant types, including full-service, was associated with higher odds of obesity. These results did not change when in a sensitivity analysis they adjusted their models for the presence of healthier food options such as supermarkets and fruit and vegetable shops ${ }^{(20)}$.

Although more sophisticated measures of exposure might, theoretically, get closer to better representing the 
residential food environment - with stronger correlations to dietary outcomes - this was not confirmed by the present study. A study using a similar complex measure of exposure to test the association between spatial access to fast-food outlets and weight status also did not find significant associations ${ }^{(13)}$. Even though we used more comprehensive measures of exposure, given all the other factors that influence the relationship between the environment and behaviour, we may not have been able to capture the complexity of the relationships by which the food environment influences the dietary patterns of individuals. The difficulty of representing exposure to the food environment and its association with health behaviours has been reported before ${ }^{(8,23,43)}$. It has been proposed that different neighbourhood definitions and multiple neighbourhood contexts (e.g. social, food and physical activity environment) should be taken into account while trying to model how exposure to the environment might influence health outcomes ${ }^{(44)}$. However, there is no consensus in the literature on what the best exposure measure would be and due to the particularities of each research setting, reaching consensus may not be possible or even appropriate. Nevertheless, the researchers' attempt on working towards the best possible representation of the food environment, within each reality, and ways to better characterize individual exposure, should always be reported and acknowledged ${ }^{(43)}$.

Some limitations of the current study need to be addressed. First, the dietary pattern measures were based on a series of basic food frequency questions, and this measure may not have been sufficiently sensitive to detect all meaningful associations. The use of crude dietary data has been reported as a common limitation and one of the potential explanations for the inconsistency found in food environment research ${ }^{(45)}$. Another potential limitation of the present study, as well as most of the previous studies, is that by focusing on the food environment around individuals' homes, we may have failed to include other relevant food environments, such as those in and around work or leisure locations. Dietary habits might be influenced by individuals' ethnic and socio-economic background. Unfortunately, due to ethical constrains, we were not able to collect information on ethnicity and income in two countries in our study. We have adjusted our models for relevant individual- and neighbourhood-level variables, including education, household composition and self-selection. We sampled the neighbourhoods based on neighbourhood SES and density and we performed adjustment for country. However, as in most observational studies, some level of residual confounding might have occurred. Strengths of the present study include the fact that it contributes to further explorations of differences between relative and absolute exposure measures of the food environment and the fact that we used a comprehensive statistical approach guided by explicit hypotheses; we accounted for the clustered nature of our data and sought to overcome potential bias due to missing data by performing multiple imputation. Finally, our large sample size, collected from five European countries, contributes to the external validity of our findings. Even though the low response rate might have produced a selective sample, the distribution of participants was well balanced in terms of sociodemographic characteristics such as percentage of males and females, level of education and BMI. Therefore, although it is not possible to exclude the possibility of residual biases within this sample, this is an indication that the sample is broadly representative of the population regarding these characteristics.

\section{Conclusions}

We examined different measures of exposure to explore the broader residential food environment and its association with dietary patterns. We could not confirm our hypotheses that more complex absolute measures or relative measures of exposure to food retailers would produce stronger associations with dietary patterns. We had some indication that absolute and relative measures of exposure assess different aspects of the food environment and this might be reflected in the direction of associations. However, given the lack of significant findings, this needs to be further explored.

\section{Acknowledgements}

Acknowledgements: The authors would like to thank all participants who took part in the SPOTLIGHT study and the members of the WP3 SPOTLIGHT group who are not co-authors of this paper, namely Célina Roda, Greet Cardon, Ilse De Bourdeaudhuij, Ketevan Glonti, Maher BenRebah, Martin McKee, Roza Adany and Thierry Feuillet. Financial support: The SPOTLIGHT project was funded by the Seventh Framework Programme (CORDIS FP7) of the European Commission, HEALTH (FP7-HEALTH-2011two-stage) (grant number 278186). M.G.M.P. has a grant from the Brazilian higher education agency CNPq (National Council for Scientific and Technological Development) as part of the Science Without Borders Program (process number 233850/2014-7). J.D.M.'s work was funded by the Netherlands Organisation for Scientific Research (NWO) with a VENI grant on 'Making the healthy choice easier - role of the local food environment' (grant number 451-17-032). H.R.'s contribution to this research was supported by the National Institute for Health Research (NIHR) Collaboration for Leadership in Applied Health Research and Care North Thames at Bart's Health NHS Trust (NIHR CLAHRC North Thames). The funding agencies cited here, namely NHS, NIHR, the Department of Health and Social Care, the European Commission, $\mathrm{CNPq}$ and NWO, had no role in the design, analysis 
or writing of this article. Conflict of interest: None. Authorship: The WP3 SPOTLIGHT group (J.D.M., J.-M.O., H.C., H.B., H.R., S.C., J.B. and J.L.) contributed to study design, research protocol and conducted the data collection. M.G.M.P. performed the data analysis and drafted the manuscript supervised by J.D.M., J.W.J.B., J.B. and J.L. All authors read, provided feedback and approved the final submitted version of the manuscript. Ethics of buman subject participation: Local ethics committees in each participating country approved the study. All survey participants provided informed consent.

\section{Supplementary material}

To view supplementary material for this article, please visit https://doi.org/10.1017/S1368980018003063

\section{References}

1. Monteiro CA, Moubarac JC, Cannon G et al. (2013) Ultraprocessed products are becoming dominant in the global food system. Obes Rev 14, Suppl. 2, 21-28.

2. Vandevijvere S, Chow CC, Hall KD et al. (2015) Increased food energy supply as a major driver of the obesity epidemic: a global analysis. Bull World Health Organ 93, 446-456.

3. Swinburn BA, Sacks G, Hall KD et al. (2011) The global obesity pandemic: shaped by global drivers and local environments. Lancet 378, 804-814.

4. Lakerveld J \& Mackenbach J (2017) The upstream determinants of adult obesity. Obes Facts 10, 216-222.

5. Boone-Heinonen J, Gordon-Larsen P, Kiefe CI et al. (2011) Fast food restaurants and food stores: longitudinal associations with diet in young to middle-aged adults: the CARDIA study. Arch Intern Med 171, 1162-1170.

6. Thornton LE \& Kavanagh AM (2012) Association between fast food purchasing and the local food environment. Nutr Diabetes 2, e53.

7. Zenk SN, Lachance LL, Schulz AJ et al. (2009) Neighborhood retail food environment and fruit and vegetable intake in a multiethnic urban population. Am J Health Promot 23, 255-264.

8. Caspi CE, Sorensen G, Subramanian SV et al. (2012) The local food environment and diet: a systematic review. Health Place 18, 1172-1187.

9. Bivoltsis A, Cervigni E, Trapp G et al. (2018) Food environments and dietary intakes among adults: does the type of spatial exposure measurement matter? A systematic review. Int J Health Geogr 17, 19.

10. Maguire ER, Burgoine T, Penney TL et al. (2017) Does exposure to the food environment differ by socioeconomic position? Comparing area-based and person-centred metrics in the Fenland Study, UK. Int J Health Geogr 16, 33.

11. Charreire H, Casey R, Salze P et al. (2010) Measuring the food environment using geographical information systems: a methodological review. Public Health Nutr 13, 1773-1785.

12. Salze P, Banos A, Oppert JM et al. (2011) Estimating spatial accessibility to facilities on the regional scale: an extended commuting-based interaction potential model. Int J Health Geogr 10, 2.

13. Mackenbach JD, Charreire H, Glonti K et al. (2018) Exploring the relation of spatial access to fast food outlets with body weight: a mediation analysis. Environ Behav. Published online: 1 January 2018. https://doi.org/ $10.1177 / 0013916517749876$.

14. Lucan SC (2015) Concerning limitations of foodenvironment research: a narrative review and commentary framed around obesity and diet-related diseases in youth. J Acad Nutr Diet 115, 205-212.

15. Ni Mhurchu C, Vandevijvere S, Waterlander W et al. (2013) Monitoring the availability of healthy and unhealthy foods and non-alcoholic beverages in community and consumer retail food environments globally. Obes Rev 14, Suppl. 1, 108-119.

16. Clary CM, Ramos Y, Shareck M et al. (2015) Should we use absolute or relative measures when assessing foodscape exposure in relation to fruit and vegetable intake? Evidence from a wide-scale Canadian study. Prev Med 71, 83-87.

17. Mason KE, Bentley RJ \& Kavanagh AM (2013) Fruit and vegetable purchasing and the relative density of healthy and unhealthy food stores: evidence from an Australian multilevel study. J Epidemiol Community Health 67, 231-236.

18. Mulrooney T, McGinn C, Branch B et al. (2017) A new raster-based metric to measure relative food availability in rural areas: a case study in Southeastern North Carolina. Southeastern Geographer 57, 151-178.

19. Zenk SN, Powell LM, Rimkus L et al. (2014) Relative and absolute availability of healthier food and beverage alternatives across communities in the United States. Am J Public Health 104, 2170-2178.

20. Polsky JY, Moineddin R, Dunn JR et al. (2016) Absolute and relative densities of fast-food versus other restaurants in relation to weight status: does restaurant mix matter? Prev Med 82, 28-34.

21. Centers for Disease Control and Prevention (2011) Census Tract Level State Maps of the Modified Retail Food Environment Index (mRFEI). Children's Food Environment State Indicator Report, 2011. Atlanta, GA: CDC.

22. Aggarwal A, Cook AJ, Jiao J et al. (2014) Access to supermarkets and fruit and vegetable consumption. Am J Public Health 104, 917-923.

23. Clary C, Lewis DJ, Flint E et al. (2016) The local food environment and fruit and vegetable intake: a geographically weighted regression approach in the ORiEL Study. $\mathrm{Am}$ J Epidemiol 184, 837-846.

24. Jolliffe IT (2010) Principal Component Analysis. New York: Springer.

25. Newby PK \& Tucker KL (2004) Empirically derived eating patterns using factor or cluster analysis: a review. Nutr Rev 62, $177-203$.

26. Hu FB (2002) Dietary pattern analysis: a new direction in nutritional epidemiology. Curr Opin Lipidol 13, 3-9.

27. Lakerveld J, Brug J, Bot S et al. (2012) Sustainable prevention of obesity through integrated strategies: the SPOTLIGHT project's conceptual framework and design. BMC Public Health 12, 793.

28. Lakerveld J, Ben Rebah M, Mackenbach JD et al. (2015) Obesity-related behaviours and BMI in five urban regions across Europe: sampling design and results from the SPOTLIGHT cross-sectional survey. BMJ Open 5, e008505.

29. Bethlehem JR, Mackenbach JD, Ben-Rebah M et al. (2014) The SPOTLIGHT virtual audit tool: a valid and reliable tool to assess obesogenic characteristics of the built environment. Int J Health Geogr 13, 52.

30. Woodruff RC, Raskind IG, Harris DM et al. (2018) The dietary impact of introducing new retailers of fruits and vegetables into a community: results from a systematic review. Public Health Nutr 21, 981-991.

31. Kant AK \& Graubard BI (2018) A prospective study of frequency of eating restaurant prepared meals and subsequent 9 -year risk of all-cause and cardiometabolic mortality in US adults. PLoS One 13, e0191584. 
32. Lachat C, Nago E, Verstraeten R et al. (2012) Eating out of home and its association with dietary intake: a systematic review of the evidence. Obes Rev 13, 329-346.

33. Penney TL, Jones NRV, Adams J et al. (2017) Utilization of away-from-home food establishments, dietary approaches to stop hypertension dietary pattern, and obesity. Am J Prev Med 53, e155-e163.

34. Richardson AS, Meyer KA, Howard AG et al. (2015) Multiple pathways from the neighborhood food environment to increased body mass index through dietary behaviors: a structural equation-based analysis in the CARDIA study. Health Place 36, 74-87.

35. Rummo PE, Guilkey DK, Shikany JM et al. (2017) How do individual-level sociodemographics and neighbourhoodlevel characteristics influence residential location behaviour in the context of the food and built environment? Findings from 25 years of follow-up in the CARDIA Study. $J$ Epidemiol Community Health 71, 261-268.

36. Moons KGM, Donders RART, Stijnen T et al. (2006) Using the outcome for imputation of missing predictor values was preferred. J Clin Epidemiol 59, 1092-1101.

37. Rubin DB (1987) Multiple Imputation for Non-Response in Surveys. New York: John Wiley \& Sons.

38. Bodner TE (2008) What improves with increased missing data imputations? Struct Equ Modeling 15, 651-675.

39. Hubbard AE, Ahern J, Fleischer NL et al. (2010) To GEE or not to GEE: comparing population average and mixed models for estimating the associations between neighborhood risk factors and health. Epidemiology 21, 467-474

40. Lamichhane AP, Warren J, Puett R et al. (2013) Spatial patterning of supermarkets and fast food outlets with respect to neighborhood characteristics. Health Place $\mathbf{2 3}$, 157-164

41. Mercille G, Richard L, Gauvin L et al. (2012) Associations between residential food environment and dietary patterns in urban-dwelling older adults: results from the VoisiNuAge study. Public Health Nutr 15, 2026-2039.

42. Rummo PE, Meyer KA, Boone-Heinonen J et al. (2015) Neighborhood availability of convenience stores and diet quality: findings from 20 years of follow-up in the coronary artery risk development in young adults study. Am J Public Health 105, e65-e73.

43. Burgoine T, Alvanides S \& Lake AA (2013) Creating 'obesogenic realities'; do our methodological choices make a difference when measuring the food environment? Int $J$ Health Geogr 12, 33.

44. Myers CA, Denstel KD \& Broyles ST (2016) The context of context: examining the associations between healthy and unhealthy measures of neighborhood food, physical activity, and social environments. Prev Med 93, 21-26.

45. Kirkpatrick SI, Reedy J, Butler EN et al. (2014) Dietary assessment in food environment research: a systematic review. Am J Prev Med 46, 94-102. 